\title{
50.
}

\section{SUR QUELQUES THÉORÈMES DE LA GÉOMÉTRIE DE POSITION.}

[From the Journal für die reine und angewandte Mathematik (Crelle), tome xxxi. (1846), pp. 213-227.]

EN prenant pour donné un système quelconque de points et de droites, on peut mener par deux points donnés des nouvelles droites, ou trouver des points nouveaux, savoir les points d'intersection de deux des droites données; et ainsi de suite. On obtient de cette manière un nouveau système de points et de droites, qui peut avoir la propriété que plusieurs des points sont situés dans une même droite, ou que plusieurs des droites passent par le même point; ce qui donne lieu à autant de théorèmes de géométrie de position. On a déjà étudié la théorie de plusieurs de ces systèmes; par exemple de celui de quatre points; de six points, situés deux à deux sur trois droites qui se rencontrent dans un même point; de six points trois à trois sur deux droites, ou plus généralement, de six points sur une conique (ce dernier cas, celui de l'hexagramme mystique de Pascal, n'est pas éncore épuisé; nous y reviendrons dans la suite), et même de quelques systèmes dans l'espace. Cependant il existe des systèmes plus généraux que ceux qui ont été examinés, et dont les propriétés peuvent être aperçues d'une manière presque intuitive, et qui, à ce que je crois, sont nouveaux. Commençons par le cas le plus simple. Imaginons un nombre $n$ de points situés d'une manière quelconque dans l'espace, et que nous désignerons par $1,2,3, \ldots n$. Qu'on fasse passer par toutes les combinaisons de deux points des droites, et par toutes les combinaisons de trois points des plans; puis coupons ces droites et ces plans par un plan quelconque, les droites selon des points, et les plans selon des droites. Soit $\alpha \beta$ le point qui correspond à la droite menée par les deux points $\alpha, \beta$; soit de même $\beta \gamma$ le point qui correspond à celle menée par les points $\beta$, $\gamma$, et ainsi de suite. Soit de plus $\alpha \beta \gamma$ la droite qui correspond au plan passant par les trois points $\alpha, \beta, \gamma$, etc. Il est clair que les trois points $\alpha \beta, \alpha \gamma, \beta \gamma$ seront situés dans la droite $\alpha \beta \gamma$. Donc en représentant par 
$N_{2}, N_{3}, \ldots$ les nombres des combinaisons de $n$ lettres prises deux à deux, trois à trois etc. à la fois, on a le théorème suivant:

Thє́овѐme I. On peut former un système de $N_{2}$ points situés trois à trois sur $N_{3}$ droites: savoir en représentant les points par 12, 13,. 23, etc. et les droites par 123, etc., les points 12, 13, 23 seront situés sur la droite 123, et ainsi de suite.

Pour $n=3$, ou $n=4$, cela est tout simple; on aura trois points sur une droite; ou six points trois à trois sur quatre droites; il n'en résulte aucune propriété géométrique. Pour $n=5$ on a dix points, trois à trois sur autant de droites, savoir les points

$$
12,13,14,15,23,24,25,34,35,45
$$

et les droites

$$
123,124,125,134,135,145,234,235, \quad 245,345 .
$$

Les points $12,13,14,23,24,34$ sont les angles d'un quadrilatère quelconque ${ }^{1}$, le point 15 est tout à fait arbitraire, le point 25 est situé sur la droite passant par les points 12 et 15, mais sa position sur cette droite est arbitraire. On déterminera depuis les points 35, 45; 35 comme point d'intersection des droites passant par 13 et 15 et par 23 et 25, c'est-à-dire des droites 135 et 235, et de même 45 comme point d'intersection des lignes 145 et 245 . Les points 35 et 45 auront la propriété géométrique d'être en ligne droite avec 34, ou bien tous les trois seront dans une même droite 345 .

Étudions de plus près la figure que nous venons de former. En prenant le cinq numéros dans un ordre déterminé, par exemple dans l'ordre naturel 1, 2, 3, 4, 5, les cinq points $12,23,34,45,51$ pourront être considérés comme formant un pentagone que nous représenterons par la notation (12345). Les côtés de ce pentagone sont évidemment 123, 234, 345, 451, 512. De même les points 13, 35, 52, 24, 41 peuvent être considérés comme formant le pentagone (13524) dont les côtés sont 135, 352, 524, 241, 413. Ce pentagone est circonscrit au premier, car ses côtés passent évidemment par les angles 15, 23, 45, 12, 34 du premier: mais il est de· même inscrit à celui-ci, car ses angles sont situés respectivement dans- les côtés 123, 345, 512, 234, 451 de ce même pentagone. Donc les pentagones

$$
\text { (12345), }
$$

sont à la fois circonscrits et inscrits l'un à l'autre, donc:

Théorème II. La figure composée de dix points, trois à trois dans dix droites, peut étre considérée (même de six manières différentes) sous la forme de deux pentagones, inscrits et circonscrits l'un d̀ l'autre.

$\mathrm{Ou}$ encore

ThÉoRÈme III. Étant donné un pentagone quelconque, on peut toujours trouver un autre pentagone qui $y$ est à la fois circonscrit et inscrit. Ce second pentagone peut satisfaire à une seule condition donnée quelconque.

1 Il faut avoir égard toujours à la différence entre quadrilutère et quadrangle; chaque quadrilatère a quatre côtés et six angles, chaque quadrangle a quatre angles et six côtés. 
Si par exemple le second pentagone doit avoir un de ses angles sur un point donné d'un côté du premier, la construction se déduit tout de suite de ce qui précède.

Ces paires correspondantes de pentagones forment une figure connue. On en trouve la construction dans une note de M. [J. T.] Graves dans le Philosophical Magaisine [vol. xv. 1839], mais la même figure est encore mieux connue sous un autre point de vue. En effet, considérons le point 12, et les droites $123,124,125$ qui passent par ce point; puis les triangles dont les angles sont $13,14,15$ et $23,24,25$. Les côtés de ces mêmes triangles sont $134,135,145$ et $234,235,245$, et les côtés correspondants se rencontrent dans les points 34, 35, 45 qui sont en ligne droite. Donc le théorème sur les pentagones est le suivant:

"Si les angles de deux triangles sont situés deux à deux dans trois droites qui se rencontrent dans un point, leurs côtés homologues se coupent dans trois points en ligne droite."

Remarquons aussi que ce théorème particulier (en n'empruntant rien des trois dimensions de l'espace) reproduit le théorème général relatif au nombre $n$. Il n'y a pour cela qu'à considérer $n$ droites passant par le même point, et qui peuvent être désignées par 1, 2, 3, . n. En choisissant d'abord les points 12, 13, tout triangle dont les trois angles sont situés dans les droites $1,2,3$, pendant que deux de ses côtés passent par 12, 13, a la propriété que le troisième côté passe par un point déterminé 23 situé dans la droite passant par 12, 13. En prenant arbitrairement le point 14, on obtient avec les droites 1, 3, 4 ou 1, 2, 4 les nouveaux points 34, 24 qui sont en ligne droite avec 23 , et ainsi de suite.

Passons au cas $n=6$. Il existe ici quinze points situés trois à trois sur vingt droites, ou bien vingt droites qui se coupent quatre à quatre en quinze-points. Il n'y a point ici des systèmes d'hexagones, mais il existe un système de neuf points qui est assez remarquable. Divisons d'une manière quelconque les numéros $1,2,3,4,5,6$ en deux suites par trois, par exemple en $1,3,5$ et 2, 4, 6, et considérons les neuf points

$$
\begin{array}{lll}
12, & 14, & 16, \\
32, & 34, & 36, \\
52, & 54, & 56 .
\end{array}
$$

Les droites qui passent par 12 et 32,14 et 34,16 et 36 , savoir $132,134,136$, se rencontrent dans le même point 13. De même les droites qui passent par 32 et 52,34 et 54,36 et 56 se rencontrent dans 35 , et les droites qui passent par 12 et 52,14 et 54 , 16 et 56 se rencontrent dans 15 . Les points 13,15 et 35 sont sur la même droite 135 . En considérant les points 12, 14, 16 comme formant un triangle, et de même les points $32,34,36$ et $52,54,56$, cela revient à dire que les droites menées par les angles homologues des triangles prises deux à deux, se rencontrent trois à trois dans trois points situés dans la même droite. Ou bien, ce que l'on savait déjà par le théorème 3: les côtés homologues des triangles se rencontrent trois à trois dans trois points situés en ligne droite. En effet, les côtés des triangles sont 124, 126, 146 pour la première, et $324,326,346$ et $524,526,546$ pour les deux autres. Les trois premiers côtés se 
rencontrent dans 24, les autres dans 26 et 46 , et ces trois points sont dans la droite 246. Maintenant tout cela arrive également en combinant les colonnes verticales, ou en considérant les neuf points comme formant les trois autres triangles dont les angles sont $12,32,52 ; 14,34,54 ; 16,36,56$. Cela donne lieu au théorème suivant:

THÉORÈme IV. Le système de quinze points, situés trois à trois sur vingt droites, contient (et cela même de dix manières différentes) un système de neuf points qui ont la propriété de former de deux manières différentes trois triangles, tels, que les droites qui passent par leurs angles homologues, prises deux à deux, se rencontrent dans trois points qui sont en ligne droite, tandis que les côtés homologues des triangles se coupent trois à trois en trois autres points qui sont aussi en ligne droite. Dans la seconde manière de former les triangles, ces deux systèmes de trnis points en ligne droite sont seulement échangés.

Il ne reste qu'à savoir combien il y en a d'arbitraires dans le système de quinze points situés trois à trois sur vingt droites. En supposant le système formé pour le nombre cinq, on peut prendre arbitrairement 16 et 26 sur la droite 126 qui est déterminée par les points 12 et 16 . Donc $12,13,14,15$ et 16 sont arbitraires et 23, 24, 25, 26 sont arbitrairement situés sur des droites données. L'existence des droites 345, $346,356,456$ constitue autant de théorèmes géométriques; c'est-à-dire, chacune de ces droites est déterminée par trois points.

En essayant d'approfondir la théorie de six points sur la même conique, on rencontrera un système de neuf points, tel que ceux que nous venons d'examiner; mais il est moins général. Il existe des relations entre les points qui n'ont pas lieu dans le système général. Je renvoie cette discussion à une section séparée de ce mémoire, et je passe au cas de $n=7$.

Pour ce cas on a tout de suite le théorème suivant:

ThÉonème V. Le système de vingt et un points situés trois à trois sur trente-cinq droites, peut être considéré (même de cent vingt manières différentes) comme composé de trois heptagones, le premier circonscrit au second, le second au troisième et le troisième au premier. Les heptagones par exemple peuvent être (12345̌67), (1357246), (1526374).

Dans ce système $12,13,14,15,16,17$ sont arbitraires, et $23,24,25,26,27$ le sont sur des droites données; les droites $345,346,347,356,357,367,456,457,467,567$ sont déterminées chacune par trois points. Dans le cas général $12,13 \ldots 1 n$ sont arbitraires, et $23 \ldots 2 n$ le sont sur des droites données. Il existe $\frac{1}{6}(n-2)(n-3)(n-4)$ droites dont chacune est déterminée par trois points. Un théorèrne analogue à celui-ci a lieu quand $n$ est un nombre premier: savoir le suivant:

THÉonÈme VI. Le système de $N_{2}$ points, situés trois à trois sur $N_{3}$ droites, peut être considéré (même de $\frac{1.2 \ldots(n-2)}{n-1}$ manières) comme composé de $\frac{1}{2}(n-1)$ n-gones, le premier circonscrit au second, le second au troisième, etc., et le dernier au premier. 
Je ne connais pas d'autres cas où l'idée des nombres premiers se présente dans la géométrie. Il sera peut-être possible de trouver des théorèmes analogues à III, IV, $\mathrm{V}$, pour toutes les formes du nombre $n$, mais je n'ai pas encore examiné cela.

Le théorème général I, peut être considéré comme l'expression d'un fait analytique, qui doit également avoir lieu en considérant quatre coordonnées au lieu de trois. Ici une interprétation géométrique a lieu, qui s'applique aux points dans l'espace. On peut en effet, sans recourir d̀ aucune notion métaphysique d̀ l'égard de la possibilité de l'espace à quatre dimensions, raisonner comme suit (tout cela pourra aussi être traduit facilement en langue purement analytique): En supposant quatre dimensions de l'espace, il faudra considérer des lignes déterminées par deux points, des demi-plans déterminés par trois points, et des plans déterminés par quatre points; (deux plans se coupent alors suivant un demi-plan, etc.). L'espace ordinaire doit être considéré comme plan, et il coupera un plan selon un plan ordinaire, un demi-plan selon une ligne ordinaire, et une ligne selon un point ordinaire. Tout cela posé : en considérant un nombre $n$ de points, et les combinant deux à deux, trois à trois, et quatre à quatre par des lignes, des demi-plans et des plans, puis coupant le système par l'espace considéré comme plan, on obtient le théorème suivant de géométrie à trois dimensions:

THÉORÈme VII. On peut former un système de $N_{2}$ points, situés trois à trois dans $N_{3}$ droites qui elles-mêmes sont situées quatre $\dot{a}$ quatre dans $N_{4}$ plans. En représentant les points par 12, 13, etc., les points situés dans la même droite sont 12, 13, 23; et les droites étant représentées par 123 etc. comme auparavant, les droites 123, 124, 134, 234 sont situées dans le même plan 1234.

En coupant cette figure par un plan, on obtient le théorème suivant de géométrie plane :

THÉORÈME VIII. On peut former un système de $N_{3}$ points situés quatre à quatre dans $N_{4}$ droites. Les points doivent être représentées par la notation 123, etc. et les droites par 1234, etc. Alors 123, 124, 134, 234 sont dans la même droite désignée par 1234.

De même, en considérant un espace à $p+2$ dimensions, on obtient la proposition suivante, encore plus générale:

ThÉoRغ̀Me IX. On peut former dans l'espace un système de $N_{p}$ points, qui passent $p+1 \grave{a} p+1$ par $N_{p+1}$ droites, situées $p+2 \grave{d} p+2$ dans $N_{p+2}$ plans, ou bien pour la géométrie plane, un système de $N_{p}$ points, situés $p+1 \grave{a} p+1$ dans $N_{p+1}$ droites.

Des théorèmes analogues à $\mathrm{IV}$ et $\mathrm{V}$ seraient probablement très nombreux et très compliqués.

Les réciproques polaires auront évidemment lieu pour tous ces théorèmes; on pourrait aussi les démontrer directement d'une manière analogue.

C. 


\section{SUR LE THÉORÈME DE PASCAL.}

En considérant six points sur la même conique, et les prenant dans un ordre déterminé, pour en former un hexagone, on sait que les côtés opposés se rencontrent dans trois points situés en ligne droite. En prenant les points dans un ordre quelconque, on en peut former soixante hexagones, à chacun desquels correspond une droite; il s'agit maintenant de trouver les relations entre ces droites.

M. Steiner a prouvé dans son ouvrage Systemutische Entwickelungen u. s. w. [1832], que ces soixante droites passent trois à trois par vingt points, et il ajoute que ces vingt points sont situés quatre à quatre sur quinze droites. La première partie de ce théorème peut être démontrée assez facilement, comme nous le verrons: mais pour la seconde partie, je n'ai pas réussi à trouver les combinaisons de quatre points qui doivent être situés en ligne droite, et il me paraît même qu'il est impossible de les trouver ${ }^{1}$.

Cherchons les combinaisons des droites qui doivent passer trois à trois par le même point.

Soient 1, 2, 3, 4, 5, 6 les six points situés sur la même conique. Considérons d'abord l'hexagone 123456 que l'on obtient en prenant les points dans un ordre déterminé. Suivant le théorème de Pascal les trois points

$$
12.45, \quad 23.56, \quad 34.61
$$

(où 12.45 désigne le point d'intersection des lignes passant par les points 1, 2 et $4,5)$ sont situés en ligne droite. Considérons les six hexagones

123456

1443652

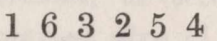

143256

1233654

1634552

qu'on tire du premier en permutant les nombres 2, 4, 6 correspondants aux sommets alternés de l'hexagone. Pour les trois premiers on fait les permutations cycliques de ces nombres (savoir 246, 462,624), pour les trois autres on fait d'abord une inversion 426,

1 Je ne sais pas s'il existe une démonstration de la seconde partie du théorème; je n'ai pu la trouver nulle part. Au cas que cette partie du théorème n'était pas correcte, il paraît que l'on devra peut-être lui substituer la proposition suivante: "Les vingt points déterminent deux à deux dix lignes qui passent trois à trois par dix points." On verra dans ce qui suit, de quelle manière il faudrait combiner ces points. [Voir 55.] 
puis les permutations cycliques $(426,264,642)$. En écrivant les combinaisons des points qui doivent être situés en ligne droite, on a

\begin{tabular}{lll}
12.45 & 23.56 & 34.61 \\
36.12 & 56.14 & 52.34 \\
45.36 & 14.23 & 16.52 \\
\hline 14.25 & 43.56 & 32.61 \\
36.14 & 56.12 & 54.42 \\
25.36 & 12.34 & 61.54
\end{tabular}

Suivant cette table les points sur la même horizontale sont en ligne droite.

On remarquera d'abord que les trois premières droites passent par les angles des triangles dont les côtés sont $36,45,12$ et 14, 23, 56. Les côtés homologues de ces triangles se rencontrent en 36.14, 45.23, 12.56 qui sont en ligne droite, c'est-à-dire, par un théorème déjà cité: les trois lignes passent par un même point. On aurait été conduit au même résultat en observant que les trois premières droites passent par les triangles dont les côtés sont 14, 23, 56 et 52, 16, 34 ou enfin 52, 16, 34 et 36, 45, 12. De même les trois dernières droites passent par le même point. Donc il a été démontré ce qui suit:

THÉonème X. En considérant les trois hexagones qu'on obtient en permutant cycliquement les angles alternés du premier, les trois droites qui y correspondent se rencontrent dans un même point. Les soixante lignes passent donc trois à trois par vingt points.

Ajoutons qu'aux trois hexägones de ce théorème correspondent d'une manière particulière trois autres hexagones, ou que les vingt points doivent se combiner deux à deux d'une manière particulière. droites

Mais on se formera une idée plus claire du système en remarquant que les neuf

$$
\begin{array}{lll}
36, & 45, & 12 \\
14, & 23, & 56 \\
25, & 61, & 34
\end{array}
$$

ont entre elles une relation qui est polaire réciproque de celle entre les neuf points du théorème IV. Pour faciliter cette comparaison, je prendrai d'abord le théorème analogue pour les tangentes d'une conique.

Théorìme XI. Soient $1,3,5$ et $2,4,6$ des tangentes à une même conique et 12 , etc. les points d'intersection de ces droites: les neuf points

$$
\begin{array}{lll}
36, & 45, & 12 \\
14, & 23, & 56 \\
25, & 61, & 34
\end{array}
$$


peuvent être déterminés au moyen de six points de l'espace $A, B, C, \alpha, \beta, \gamma$, de manière que $A \alpha$, etc. représente le point d'intersection de la droite passant par $A, \alpha$ avec le plan de la figure. Les points sont correspondants entre eux de cette manière:

$$
\begin{array}{lll}
A \alpha, & A \beta, & A \gamma \\
B \alpha, & B \beta, & B \gamma \\
C \alpha, & C \beta, & C \gamma
\end{array}
$$

seulement les points $36,23,34$ etc. sont en ligne droite, ce qui n'aurait pas lieu pour les points $A \alpha, B \beta, C \gamma$, si la position de $A, B, C, \alpha, \beta, \gamma$ était arbitraire. On est donc conduit à ce problème:

Trouver six points $A, B, C, \alpha, \beta, \gamma$ dans l'espace, tels, qu'en représentant par $A \alpha$, etc. l'intersection de la droite menée par $A \alpha$ avec un plan donné, les combinaisons des points

$$
\begin{aligned}
& (A \alpha, \quad B \beta, \quad C \gamma) \\
& (A \beta, \quad B \gamma, C \alpha) \\
& (A \gamma, B \alpha, C \beta) \\
& (A \alpha, B \gamma, C \beta) \\
& (A \beta, B \alpha, C \gamma) \\
& (A \gamma, B \beta, C \alpha) \text {. }
\end{aligned}
$$

soient en ligne droite.

Pour le théorème de Pascal, cela donne:

ThÉonìme XII. Soient 1, 3, 5 et 2, 4, 6 des points d'une conique, les neuf lignes

$$
\begin{array}{lll}
36, & 45, & 12 \\
14, & 23, & 56 \\
25, & 61, & 34
\end{array}
$$

peuvent être considérées comme les projections des lignes

$$
\begin{array}{lll}
A \alpha, & A \beta, & A \gamma \\
B \alpha, & B \beta, & B \gamma \\
C \alpha, & C \beta, & C \gamma
\end{array}
$$

sur le plan de la figure, ò̀ $A, B, C, \alpha, \beta, \gamma$ sont six plans, dont la relation reste encore d̀ déterminer.

En effectuant la solution du problème que j'ai indiquéé on aurait, à ce qu'il me semble, un point de vue tout à fait nouveau d'envisager les coniques. 
Je vais ajouter encore quelques réflexions sur la manière de chercher les relations qui existent entre les vingt points. En écrivant seulement les angles alternés des hexagones, on a cette table:

1. 2.3

1. 2.4

1. 2.5

1. 2.6

1.3 .4

1.3 .5

1.3 .6

1.4 .5

1. 4.6

1.5 .6

A chaque symbole correspondent six hexagones, qui, à ce que nous avons vu, se partagent en deux paires de trois hexagones, et à chaque combinaison de trois, il correspond un point. Il y a donc deux points qui correspondent au symbole 1.3.5, deux qui correspondent au symbole 1.3.6, deux au symbole 1.5.6 etc. En représentant donc par $\overline{35}, \overline{36}, \overline{56}$, les droites passant par ces paires de points, il me paraît probable que ces droites aient ensemble les relations du théorème $I$, (savoir que $\overline{35}, \overline{36}, \overline{56}$ se rencontrent dans un point etc.), ce qui donnerait lieu au théorème hypothétique que j'ai énoncé dans une note. Voilà, à ce que je puis apercevoir, la seule manière symétrique de combiner les droites. Mais au moins les symboles

$$
\begin{aligned}
& 1.3 .5 \\
& 1.3 .6 \\
& 1.5 .6
\end{aligned}
$$

ont entre eux des rapports singuliers. En effet, écrivons pour chacun les neuf points du théorème XII, on a ce tableau :

\begin{tabular}{lll}
36, & 45, & 12 \\
14, & 23, & 56 \\
25, & 61, & 34 \\
\hline 35, & 64, & 12 \\
14, & 23, & 56 \\
26, & 15, & 34 \\
\hline 35, & 46, & 12 \\
14, & 25, & 36 \\
26, & 13, & 54
\end{tabular}

qui ne contient que quatorze points. Cela mérite des recherches ultérieures. 
Démonstration analytique du théorème de Pascal, et de la première partie de celui de M. Steiner. Formules relatives au même sujet.

Soient $P=0, Q=0, R=0$ les équations des lignes $12,34,56$. On démontrera assez facilement que les équations des lignes 45, 61, 23 peuvent être représentées par

$$
\begin{aligned}
P+\nu Q+\mu R & =0 \\
\nu P+Q+\lambda R & =0 \\
\mu P+\lambda Q+R & =0 .
\end{aligned}
$$

En effet les six points 1, 2, 3, 4, 5, 6 seront situés dans la conique

$$
P^{2}+Q^{2}+R^{2}+\lambda+\frac{1}{\lambda} Q R+\mu+\frac{1}{\mu} P R+\nu+\frac{1}{\nu} P Q=0 ;
$$

car en faisant dans cette équation $P=0$, l'équation se réduit à

$$
\frac{1}{\lambda}(Q+\lambda R)(\lambda Q+R)=0
$$

c'est-à-dire, la conique contient les points déterminés par

$$
\begin{array}{ll}
(P=0, & \nu P+Q+\lambda R=0), \\
(P=0, & \mu P+\lambda Q+R=0),
\end{array}
$$

ou bien les points 1,2 ; et de même elle contient les autres points $3,4,5,6$. Les fonctions $P, Q, R$ sont censées contenir chacune deux constantes arbitraires; donc on a neuf constantes arbitraires dans ce système, qui par conséquent est tout-à-fait général. On peut former le système suivant d'équations:

$\begin{array}{rlr}\text { 12. } & P & =0, \\ \text { 13. } & \lambda \mu P+Q+\lambda R & =0, \\ \text { 14. } \lambda P+\mu Q+\lambda \mu R & =0, \\ \text { 15. } & \lambda P+\nu Q+\nu \lambda R & =0, \\ \text { 16. } & \nu P+Q+\lambda R & =0, \\ \text { 23. } & \mu P+\lambda Q+R & =0, \\ \text { 24. } & P+\mu \lambda Q+\mu R & =0, \\ \text { 25. } & \lambda P+\nu \lambda Q+\nu R & =0, \\ \text { 26. } & \nu \lambda P+\lambda Q+\quad R & =0, \\ \text { 34. } & \\ \text { 35. } & \mu P+\mu \nu Q+\quad R & =0, \\ \text { 36. } & \mu \nu P+\mu Q+\nu R & =0, \\ \text { 45. } & P+\nu Q+\mu R & =0, \\ \text { 46. } & \nu P+Q+\nu \mu R & =0, \\ \text { 56. } & R & =0 .\end{array}$


Écrivons les équations des lignes comprises dans la table de neuf points ci-dessus donnés. On a d'abord

$$
\begin{aligned}
\mu \nu P+\mu Q+\nu R=0, & P+\nu Q+\mu R=0, & P=0, \\
\lambda P+\mu Q+\lambda \mu R=0, & \mu P+\lambda Q+R=0, & R=0, \\
\lambda P+\nu \lambda Q+\nu R=0, & \nu P+Q+\lambda R=0, & Q=0 .
\end{aligned}
$$

En combinant la seconde et la troisième colonne verticale du tableau, on obtient pour les trois points d'intersection des côtés opposés de l'bexagone 123456, les équations

$$
\begin{array}{ll}
(P=0, & \mu Q+\nu R=0), \\
(R=0, & \lambda P+\mu Q=0), \\
(Q=0, & \lambda P+\nu R=0),
\end{array}
$$

qui appartiennent à trois points situés sur la droite

$$
\lambda P+\mu Q+\nu R=0,
$$

ce qui suffit pour démontrer le théorème de Pascal.

On obtient de même, en combinant les autres paires de colonnes verticales, deux systèmes de trois points, respectivement situés dans les droites

$$
\begin{aligned}
\frac{P}{\lambda}+\frac{Q}{\mu}+\frac{R}{\nu} & =0 \text { et } \\
\left(\frac{P}{\lambda}+\frac{Q}{\mu}+\frac{R}{\nu}\right) \lambda \mu \nu+\lambda P+\mu Q+\nu R & =0,
\end{aligned}
$$

lesquelles, avec la droite qu'on vient de trouver,

$$
\lambda P+\mu Q+\nu R=0,
$$

se rencontrent évidemment dans un même point, déterminé par les deux équations

$$
\begin{gathered}
\lambda P+\mu Q+\nu R=0 \text { et } \\
\frac{P}{\lambda}+\frac{Q}{\mu}+\frac{R}{\nu}=0 .
\end{gathered}
$$

Voilà une démonstration de la première partie du théorème de M. Steiner. Les équations que nous venons de trouver appartiennent au point d'intersection des trois droites qui correspondent au premier des trois hexagones du symbole 1.3.5. Pour trouver l'autre point correspondant de la même manière à ce symbole, il faut combiner les colonnes horizontales, ce qui donne pour les coordonnées de ce point:

$$
(\lambda-\mu \nu) P=(\mu-\nu \lambda) Q=(\nu-\lambda \mu) R .
$$

En cherchant de même les expressions des points qui correspondent aux symboles 1.3.6 et 1.5.6, on obtient des résultats moins élégants, mais qui valent peut-être la peine d'être énoncés ici.

Je forme cette table complète: 
Pour 1.3.5

Systèmes de trois lignes, qui se rencontrent dans un point.

I.

$$
\begin{aligned}
& \left\{\begin{array}{l}
\lambda P+\mu Q+\nu R=0, \\
\frac{P}{\lambda}+\frac{Q}{\mu}+\frac{R}{\nu}=0, \\
\lambda P+\mu Q+\nu R+\lambda \mu \nu\left(\frac{P}{\lambda}+\frac{Q}{\mu}+\frac{R}{\nu}\right)=0 ;
\end{array}\right. \\
& \left\{\begin{array}{l}
(\lambda-\mu \nu) P=(\nu-\lambda \mu) R, \\
(\nu-\lambda \mu) R=(\mu-\nu \lambda) Q, \\
(\mu-\nu \lambda) Q=(\lambda-\mu \nu) P ;
\end{array}\right.
\end{aligned}
$$

Pour 1.3.6

I.

$$
\left\{\begin{array}{l}
\mu P+\lambda Q+\lambda \mu \nu R=0, \\
\nu \lambda P+\mu \nu Q+\quad R=0, \\
(\mu P+\lambda Q+\lambda \mu \nu R)+(\nu \lambda P+\mu \nu Q+R)=0 ;
\end{array}\right.
$$

II.

$$
\left\{\begin{array}{l}
(\mu-\nu \lambda) P+(1-\mu \nu \lambda) R=0 \\
(1-\nu \lambda \mu) R+(\lambda-\nu \mu) Q=0 \\
(\lambda-\mu \nu) Q-(\mu-\nu \lambda) P=0
\end{array}\right.
$$

Pour 1.5.6

$$
\begin{aligned}
& \text { I. } \quad\left\{\begin{array}{ccr}
\left(\mu \nu-\lambda \mu^{2} \nu^{2}-\lambda+\lambda \mu^{2}\right) P+ & (\mu-\nu \lambda) Q+ & \left(\mu^{2} \nu-\lambda \mu \nu^{2}\right) R=0, \\
\left(\mu \nu-\lambda \mu^{2} \nu^{2}-\lambda+\lambda \nu^{2}\right) P+ & \left(\mu \nu^{2}-\lambda \mu^{2} \nu\right) Q+ & (\nu-\lambda \mu) R=0, \\
\left(\lambda \mu^{2}-\lambda \nu^{2}\right) P+\left(\mu-\nu \lambda-\mu \nu^{2}+\lambda \mu^{2} \nu\right) Q+\left(\mu^{2} \nu-\lambda \mu \nu^{2}-\nu+\lambda \mu\right) R=0
\end{array}\right. \\
& \text { II. }\left\{\begin{array}{ccc}
\left(\lambda-\lambda \nu^{2}+\mu \nu-\lambda \mu^{2}\right) P+ & \left(\mu-\lambda \mu^{2} \nu\right) Q+ & \left(\nu-\lambda \mu \nu^{2}\right) R=0, \\
\left(\lambda \nu^{2}-\lambda \mu^{2} \nu^{2}-\mu \nu+\lambda \mu^{2}\right) P+ & \left(\nu \lambda-\mu \nu^{2}\right) Q+ & \left(\lambda \mu-\mu^{2} \nu\right) R=0, \\
\left(\lambda-\lambda \mu^{2} \nu^{2}\right) P+\left(\nu \lambda-\mu \nu^{2}+\mu-\lambda \mu^{2} \nu\right) Q+\left(\lambda \mu-\mu^{2} \nu+\nu-\lambda \mu \nu^{2}\right) R=0 .
\end{array}\right.
\end{aligned}
$$

\section{Note sur le théorème de $\mathrm{M}$. Brianchon.}

On peut donner une démonstration semblable de ce théorème, en prenant pour les équations des six tangentes celles-ci:

$$
\begin{array}{ll}
\text { 1. } & P=0, \\
\text { 3. } & Q=0, \\
\text {.5. } & R=0, \\
\text { 4. } & \alpha P+\beta Q+\gamma R=0, \\
\text { 6. } & \alpha P+\beta^{\prime} Q+\gamma^{\prime} R=0, \\
\text { 2. } & \alpha^{\prime \prime} P+\beta^{\prime \prime} Q+\gamma^{\prime \prime} R=0,
\end{array}
$$

et en cherchant la relation entre les coefficients qui est nécessaire pour que ces six équations appartiennent aux tangentes d'une même conique. On obtient facilement

$$
\left(\alpha \gamma^{\prime}-\alpha^{\prime} \gamma\right)\left(\beta^{\prime} \alpha^{\prime \prime}-\beta^{\prime \prime} \alpha^{\prime}\right)\left(\gamma^{\prime \prime} \beta-\gamma \beta^{\prime \prime}\right)=\left(\alpha^{\prime} \gamma^{\prime \prime}-\alpha^{\prime \prime} \gamma^{\prime}\right)\left(\beta^{\prime \prime} \alpha-\beta \alpha^{\prime \prime}\right)\left(\gamma \beta^{\prime}-\gamma^{\prime} \beta\right),
$$

ce qui exprime aussi la condition pour que les trois diagonales se rencontrent dans un même point. 